\title{
The association between kidney function and efficacy of sugammadex in the reversal of neuromuscular blockade train-of-four and kidney functions
}

\begin{abstract}
Introduction and aim: Rocuronium-sugammadex complex is eliminated through kidneys. Rocuronium's action is prolonged in patients with renal failure. In comparison to patients with normal renal function, patients with severe renal damage show significantly prolonged elimination time for sugammadex or rocuroniumsugammadex complex. The present study aims to investigate whether renal functions are affected after sugammadex administration and to examine the time required to achieve a train-of-four ratio of 0.9 .
\end{abstract}

Material and methods: The study was carried out with 90 cases, ASA I, II and III aged between 18 and 65 years, undergoing general anesthesia. Patients were categorized as normal kidney function and impaired renal function. According to body mass index (BMI), patients were grouped as healthy $(<25 \mathrm{~kg} / \mathrm{m} 2)$, overweight $(25-30 \mathrm{~kg} / \mathrm{m} 2)$ and obese $(>30 \mathrm{~kg} / \mathrm{m} 2)$. Preoperative and postoperative creatinine clearance and BUN/Cre ratios were measured.

Results: According to the result of t-test for comparison of TOF, there was no statistically significant difference in mean TOF values between male and female patients in any period $(p>0.05)$. Mean TOF value of patients with a BMI of $>30$ $\mathrm{kg} / \mathrm{m} 2$ was significantly higher in comparison to patients with a BMI of $<25 \mathrm{~kg} / \mathrm{m} 2$ $(\mathrm{p}=0.001)$. Bun and Cre values showed significant reduction at the postoperative period in comparison to preoperative period $(\mathrm{p}=0.022, \mathrm{p}=0.011$, respectively). All TOF measurements showed negative correlation with preoperative and postoperative $\mathrm{CrCl}(\mathrm{p}=0.006, \mathrm{p}=0.004, \mathrm{p}<0.001, \mathrm{p}<0.001, \mathrm{p}=0.003, \mathrm{p}=0.002, \mathrm{p}<0.001$ and $\mathrm{p}<$ 0.001 respectively)

Conclusion: In patients with impaired kidney function with reduced creatinine clearance and in dehydrated cases with elevated $\mathrm{BUN} / \mathrm{Cr}$ ratio, time to achieve TOF 90 by reversal of neuromuscular blockade with sugammadex is minimally prolonged with moderate improvement in postoperative kidney functions, therefore we think it is safe to use.

Keywords: sugammadex, train-of-four, BUN/creatinine ratio, creatinine clearance
Volume 10 Issue 5 - 2018

\author{
Ebru Canakci,' Ahmet Karatas ${ }^{2}$ \\ 'Assistant Professor, Department of Anesthesiology and \\ Reanimation, Ordu University, Turkey \\ ${ }^{2}$ Assistant Professor, Department of Internal Medicine, Ordu \\ University, Division of Nephrology, Turkey
}

\begin{abstract}
Correspondence: Ebru Canakci,Assistant Professor, Department of Anesthesiology and Reanimation, Ordu University, Faculty of Medicine, Training and Research Hospital, Turkey, Tel +90 532 265I687, Fax 900452 2250190, Email canekciebru@gmail.com
\end{abstract}

Received: October 10, 2018 | Published: October 24, 2018

\begin{abstract}
Abbreviations: TOF, train of four; TOF0, time required to achieve a train-of-four ratio of 0 ; TOF 25 , time required to achieve a train-of-four ratio of $25 \%$; TOF90, time required to achieve a train-of-four ratio of $90 \%$; TOF $25-90$, time required to achieve a train-of-four ratio from $25 \%$ to $90 \%$; TOFend, train-of-four $\%$ ratio at the end of surgical procedure; $\mathrm{CrCl}$, creatinine clearance; $\mathrm{BUN}$, blood urea nitrogen; BMI, body mass index; IBW, ideal body weight; $\mathrm{BUN} / \mathrm{Cr}$ ratio, BUN/creatinine ratio; PORC, postoperative residual curarization; PACU, post-anesthesia care unit
\end{abstract}

\section{Introduction}

Currently, sugammadex is a commonly used agent for reversal of neuromuscular blockade induced by rocuronium. Rocuroniumsugammadex complex is eliminated through kidneys. Rocuronium's action is prolonged in patients with renal failure. ${ }^{1,2}$ In comparison to patients with normal renal function $(\mathrm{CrCl}>80 \mathrm{ml})$, patients with severe renal damage $(\mathrm{CrCl}<30 \mathrm{ml} / \mathrm{min})$ show significantly prolonged elimination time for sugammadex or rocuronium-sugammadex complex. However, no change in sugammadex efficacy or no neuromuscular recurarization was reported. ${ }^{1}$ Reduction of plasma sugammadex concentration after its administration in patients with severe renal damage show variation; therefore use of sugammadex in patients with severe renal damage is not recommended. ${ }^{3}$ Most patients with drug-related nephrotoxicity have underlying risk factor predisposing to the damage. Nephrotoxicity can affect any part of the kidney and this can result in one or multiple clinical kidney pathologies including acute or chronic kidney disease, various tubulopathies and kidney diseases with proteinuria. ${ }^{4}$ As parts of the kidney function tests, the ratio of BUN (= Blood Urea Nitrogen) to creatinine $(=\mathrm{Cre})$ is an important indicator for evaluating kidney function. Normal BUN/creatinine ratio (BUN/Cre ratio) ranges between 10/1 to 20/1. 
In infants, this ratio may be as high as 30/1 during the first 12 months. BUN/Cre ratio may rise in conditions like kidney failure, excessive protein diet, gastrointestinal bleeding,urinary tract obstructions dehydration and steroid therapy, whereas this ratio reduces in intrinsic renal diseases ${ }^{5}$ The effect of the use of rocuronium-sugammadex, eliminated by kidneys, in patients with elevated BUN/Cre ratio and patients with reduced creatinine clearance is not known. The present study aims to investigate whether renal functions are affected after sugammadex administration and to examine the time required to achieve a train-of-four (TOF) ratio of 0.9 .

\section{Materials and methods}

The study was approved by Clinical Research Ethics Committee of Ordu University Faculty of Medicine (Issue date 24/08/2017, issue number: 2017/93). The study was planned as a prospective, randomized study and was carried out between January 1st, 2017 and June 30th 2017 in operating rooms of Department of Anesthesiology and Reanimation in Ordu University, Faculty of Medicine. The study was conducted on 90 cases aged between 18-65 years with ASA (American Society of Anesthesiologists) I, II and III, undergoing general anesthesia.

All patients provided informed consent. Patients were categorized as normal kidney function (creatinine clearance and BUN/creatinine ratio within normal limits) and impaired renal function (creatinine clearance below $80 \mathrm{ml} / \mathrm{min}$, or BUN/creatinine ratio increased above 20/1). According to body mass index (BMI), patients were grouped as healthy $(<25 \mathrm{~kg} / \mathrm{m} 2)$, overweight $(25-30 \mathrm{~kg} / \mathrm{m} 2)$ and obese $(>30 \mathrm{~kg} /$ $\mathrm{m} 2)$. Study exclusion criteria were allergy to agents used in the study, neurological disorder or history of trauma involving the hand that will be used for neuromuscular monitoring, use of drugs that can interfere with neuromuscular blockade (anticonvulsant, aminoglycoside polypeptide antibiotics etc.), pregnancy-lactation state, hepatic or metabolic failure, Mallampati score III or above, or anticipated difficult intubation. Demographic data of patients [(age, height, weight, BMI (= Body mass index), ideal body weight (= IBW)] were recorded. BMI was calculated as weight $(\mathrm{kg}) /\left[\right.$ height $\left.(\mathrm{m})^{2}\right]$, while IBW was calculated as $22 \times$ [height $\left(\mathrm{m}^{2}\right)$ ]

Creatine clearance $(=\mathrm{CrCl})$ was calculated using the CockcraftGault formula.

Creatinine Clearance $(\mathrm{ml} / \mathrm{min})=(140$-age $) \times \mathrm{IBW} /$ Serum Cre $(\mathrm{mg} /$ dl) $\times 72$

All cases no given pre-medication. Routine hemodynamic and TOF monitoring was applied to patients transferred to the operating room. During the operation, systolic and diastolic blood pressures, heart rate, peripheral oxygen saturation, adductor muscle temperature and nasal temperature were monitored. Neuromuscular conductance was monitored using TOF-WATCH® SX (Organon, Dublin, Ireland) device. Figure 1 shows the TOF device used. The skin was cleaned with alcohol-soaked cotton and left to dry and then the distal electrode (Neotrode ${ }^{\circledR}$ Neonatal ECG Electrode, USA) was placed on the volar side of the wrist close to the trace of ulnar artery, $1 \mathrm{~cm}$ above the wrist joint, over the ulnar nerve. The proximal electrode was placed $2-3 \mathrm{~cm}$ proximal to the distal electrode. Acceleration transducer was mounted on the thumb and was fixated to the operating table with a plaster to allow free movement of the thumb. Temperature probe was fixated to the thenar region of the hand with a plaster. Patients were covered with attention given not to allow thenar region temperature drop below $32^{\circ} \mathrm{C}$. Ambient temperature in the operating room was adjusted at $21-23^{\circ} \mathrm{C}$. An intravenous line was placed and fluid replacement was initiated (Ringer lactate, $10 \mathrm{ml} / \mathrm{kg} /$ hour). All fluids given to the patients were brought to room temperature. All patients received preoxygenation with $100 \%$ oxygen. Induction anesthesia was performed with intravenous (i.v.) administration of propofol $2 \mathrm{mg} / \mathrm{kg}$ (Propofol ${ }^{\circledR}$ $1 \%$ Fresenius Kabi), fentanyl $1 \mathrm{mcg} / \mathrm{kg}$ (Fentanyl citrate ${ }^{\circledR}$ 2 $\mathrm{ml} / 100$ $\mathrm{mcg}$ ) and $1 \mathrm{mg} / \mathrm{kg}$ lidocaine (Aritmal 2\% Oselllaç Turkey). When the eyelash reflex disappeared, calibration was performed with peripheral nerve stimulator. Consequently, three control single twitch $(0.1 \mathrm{~Hz})$ values were obtained after 1 minute of waiting. Then, 3 control TOF values were obtained. Afterwards, rocuronium (Esmeron ${ }^{\circledR} 50 \mathrm{mg} / 5$

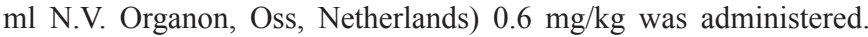
The interval between rocuronium administration to TOF0, zero TOF value, was recorded. Patients were intubated when TOF value was zero. In all patients, maintenance of anesthesia was provided with $50 / 50 \%$ O2-N2O and 2\% sevoflurane. During the surgery, when TOF value reached $25 \%$, additional rocuronium with $1 / 4$ of the intubation dose was administered and the time was recorded as TOF25. For all patients, total rocuronium dose was recorded. At the end of the surgical procedure, TOF value was recorded as TOF end. Surgical time was recorded as the interval from initiation of surgical incision until the last suture at the end of the operation. In case TOF value at the end of the operation did not reach $25 \%$, additional time was given to reach $25 \%$. After the end of the surgery, when TOF value was $25 \%$, sugammadex $2 \mathrm{mg} / \mathrm{kg}$ was administered i.v to reverse the non-depolarizing blockade. The time required to achieve a TOF rate from $25 \%$ to $90 \%$ following sugammadex administration was recorded as TOF25-90. Once TOF value reached 90\%, sevoflurane inhaler gas was shut down. Patients were extubated when respiratory rate was $>8 / \mathrm{min}, \mathrm{EtCO} 2$ value was $<50 \mathrm{mmHg}$, $\mathrm{SpO} 2$ value was $>90 \%$ and adequate tidal volume was established. The interval between TOF90 value and extubation of patients was defined as extubation time. Following extubation, patients were transferred to postoperative care unit $(=\mathrm{PACU})$ once their overall condition was stabilized. For monitoring postoperative curarization $(=\mathrm{PORC})$ in PACU, patients were assessed with head-raise and tongue stick out tests every three minutes for a total of 30 minutes. After admission to PACU, patients were monitored for at least 30 minutes and those who did not show PORC and had Aldrete scores $\geq 9$ were transferred to the ward. The intraoperative fluid requirement was calculated based on ideal body weight, extent of surgical area and fasting times and fluid replacement was administered accordingly. Patients were allowed to drink water or liquids until 2 hours prior to operation with consideration of preoperative fasting duration. Preoperative biochemical tests were analyzed one day before the operation, whereas postoperative biochemical tests were analyzed 24 hours after the operation. Some of the cases were hospitalized 48 hours postoperatively and others were admitted for 24 hours. Therefore, control biochemistry values of all cases were taken after 24 hours. Since no invasive procedure was performed, no renal biopsy was performed. The flow chart of our work is shown in Figure 2. 


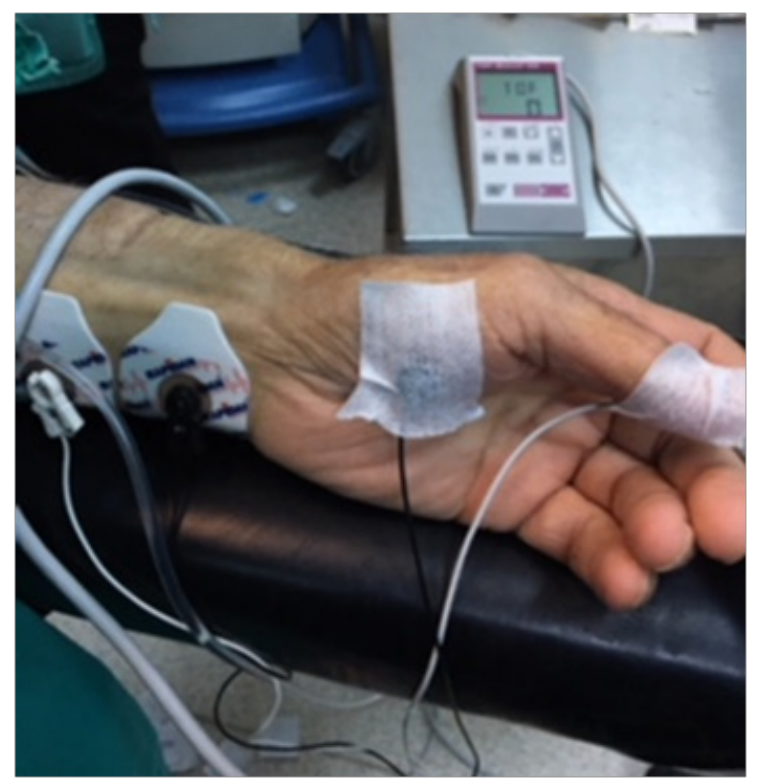

Figure I The TOF device used for neuromuscular transmission monitoring.

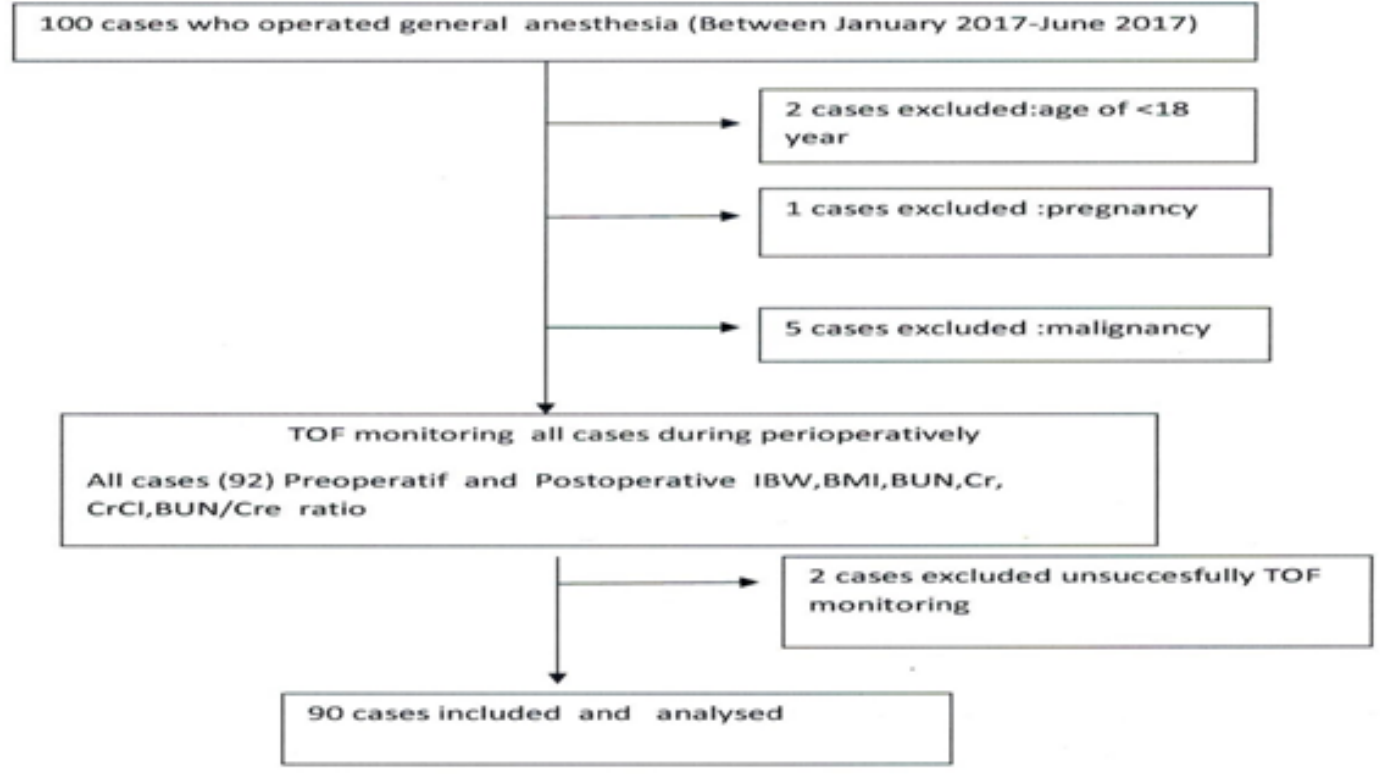

Figure 2 Flow diagram of inclusions and exclusions for patients.

\section{Statistical analysis}

Normality assessment of the data was made using KolmogorovSmirnov test. Homogenity of group variances was analyzed with Levene test. Descriptive statistics values such as mean, standard error and standard deviation were calculated for study variables. Comparison of two dependent groups (before-after) was made with paired t-test. Comparison of two independent groups (female-male) was made with student t-test. Comparison of TOF0, TOF25, TOF2590 and TOF end between more than two dependent groups was made with repeated measures variance analysis (one-way repeated ANOVA). More than two independent groups (BMI groups) were compared with one-way analysis of variance (ANOVA). In case it was required after variance analysis, different means were identified with (Tukey multiple-comparison test). Correlation between variables was determined with correlation analysis and Pearson correlation coefficients were calculated separately for each group. The level of significance $(\alpha)$ in statistical analyses and for interpretation of results was accepted as 5\%. All calculations were performed using SPSS v24 (IBM Inc., Chicago, IL, USA) statistical package program. 


\section{Results}

Descriptive statistics and comparison results for age, weight, height, IBW, difference from IBW, BMI, operation time and total amount of rocuronium according to gender shown in Table 1. There was no statistically significant difference between male and female patients regarding age, BMI, or total rocuronium amount $(\mathrm{p}>0.05)$. According to the results of $t$ test conducted for IBW, mean IBW was significantly higher in males compared to females $(p<0.001)$. Mean height and mean weight were significantly higher in males compared to females ( $p<0.001$ and $p=0.010$, respectively). Mean operation time and mean difference from IBW were significantly higher in females compared to males $(\mathrm{p}=0.011$ and $\mathrm{p}=0.010$, respectively). Descriptive statistics and comparison results for TOF according to genders and periods shown are in Table 2 .

According to the result of t-test for comparison of TOF, there was no statistically significant difference in mean TOF values between male and female patients in any period $(\mathrm{p}>0.05)$. The difference between periods was compared with (one-way Repeated ANOVA) and the difference was found to be significant $(\mathrm{p}<0.001)$. Tukey multiple comparison test was performed to identify the different means and the results were given in letters alongside the means. Tukey test results did not show difference between TOF25, TOF25-90 and TOF end times, while initial TOF value was found to be significantly lower in comparison to TOF25-90 and final TOF time $(\mathrm{p}<0.05)$. Descriptive statistics and comparison results for TOF according to BMI groups are shown in Table 3

Whether TOF differed across the BMI groups was analyzed with one-way ANOVA. The results showed that TOF did not differ in 0 , 25 and 25-90 periods according to BMI groups $(\mathrm{p}>0.05)$. TOF end time, however, showed change between BMI subsets $(\mathrm{p}<0.01)$ and different means were identified with Tukey multiple comparison test. According to the results of Tukey test, mean TOF value was significantly higher in patients with BMI $>30 \mathrm{~kg} / \mathrm{m} 2$ in comparison to those with BMI $<25 \mathrm{~kg} / \mathrm{m} 2$ ( $\mathrm{p}=0.001)$. Descriptive statistics and comparison results for BUN, Bun/Cre ratio, Cre and $\mathrm{CrCl}$ according to the genders and TOF periods are shown in Table 4.

$\mathrm{CrCl}$ levels were compared with t-test, accordingly, mean $\mathrm{CrCl}$ was higher in males compared to females both at preoperative and postoperative periods $(\mathrm{p}=0.020, \mathrm{p}=0.002$, respectively). $\mathrm{BUN}, \mathrm{BUN} /$ Cre ratio and Cre did not show asignificant difference between the sexes $(p>0.05)$. The periods were compared with paired $t$-test and $\mathrm{CrCl}$ did not show significant difference between the periods $(\mathrm{p}>0.05)$. The same was also valid for BUN/Cre ratio, whereas BUN and Cre levels showed significant reduction at the postoperative period compared to the preoperative values $(\mathrm{p}=0.022, \mathrm{p}=0.011$, respectively). Descriptive statistics and comparison results for $\mathrm{BUN}, \mathrm{BUN} / \mathrm{Cre}$ ratio, Cre and $\mathrm{CrCl}$ according to the BMI groups shown in Table 5. Whether $\mathrm{BUN}, \mathrm{BUN} / \mathrm{Cre}$ ratio, $\mathrm{Cre}$ and $\mathrm{CrCl}$ differed between $\mathrm{BMI}$ groups was analyzed with one-way ANOVA test. Accordingly, none of these parameters showed difference according to BMI groups both at the preoperative or postoperative periods $(\mathrm{p}>0.05)$. Correlation matrix between TOF variables and preoperative and postoperative renal variables are shown in Table 6.

All TOF variables showed very strong correlation with preoperative Cre values $(\mathrm{p}<0.001, \mathrm{p}<0.001, \mathrm{p}<0.001, \mathrm{p}<0.001$, respectively $)$. In other words, as Cre values increase, TOF times become longer. Similarly, all TOF measurements showed very strong correlation with postoperative Cre values ( $\mathrm{p}<0.001, \mathrm{p}<0.001, \mathrm{p}<0.001, \mathrm{p}<0.001$, respectively). There were negative correlations between all TOF measurements and preoperative $\mathrm{CrCl}(\mathrm{p}=0.006, \mathrm{p}=0.004, \mathrm{p}<0.001$, $\mathrm{p}<0.001$, respectively). Similarly, all TOF measurements showed negative correlation with postoperative $\mathrm{CrCl}(\mathrm{p}=0.003, \mathrm{p}=0.002, \mathrm{p}<$ 0.001 and $p<0.001$, respectively). In other words, in patients with impaired renal function with low creatinine clearance values and in dehydrated cases with increased BUN/Cre ratio, the time required to achieve TOF90 by reverse- acting potency of sugammadex was minimally prolonged.

Table I Descriptive statistics and comparison results for age, weight, height, IBW, difference from IBW, BMI, operation time and total amount of rocuronium according to gender

\begin{tabular}{|c|c|c|c|c|c|c|c|}
\hline Variables & Sex & $\mathbf{n}$ & Mean & Std. Dev. & Std. Error & t-Value & P-Value \\
\hline \multirow{2}{*}{ Year } & female & 43 & 47.791 & $|5.17|$ & 2.314 & \multirow{2}{*}{1.895} & \multirow{2}{*}{0.061} \\
\hline & male & 47 & 41.936 & 14.137 & 2.062 & & \\
\hline \multirow{2}{*}{ weight } & female & 43 & 76.070 & 13.793 & 2.103 & \multirow{2}{*}{-2.634} & \multirow{2}{*}{$0.010 * *$} \\
\hline & male & 47 & 83.957 & 14.545 & 2.122 & & \\
\hline \multirow{2}{*}{ height } & female & 43 & 1.603 & 0.066 & 0.010 & \multirow{2}{*}{-8.442} & \multirow{2}{*}{$0.000 * * *$} \\
\hline & male & 47 & 1.729 & 0.074 & 0.011 & & \\
\hline \multirow{2}{*}{ IBW } & female & 43 & 52.744 & 6.110 & 0.932 & \multirow{2}{*}{-11.143} & \multirow{2}{*}{$0.000 * * *$} \\
\hline & male & 47 & 68.319 & 7.059 & 1.030 & & \\
\hline \multirow{2}{*}{ Differ =weight-IBW } & female & 43 & 23.326 & 13.766 & 2.099 & \multirow{2}{*}{2.626} & \multirow{2}{*}{$0.010 * * *$} \\
\hline & male & 47 & 15.638 & 13.965 & 2.037 & & \\
\hline \multirow{2}{*}{ BMI } & female & 43 & 29.641 & 5.390 & 0.822 & \multirow{2}{*}{1.445} & \multirow{2}{*}{0.152} \\
\hline & male & 47 & 28.100 & 4.719 & 0.688 & & \\
\hline \multirow{2}{*}{ Operation Time } & female & 43 & 1.06 .372 & 54.159 & 8.259 & \multirow{2}{*}{2.592} & \multirow{2}{*}{$0.011 *$} \\
\hline & male & 47 & 77.404 & 51.828 & 7.560 & & \\
\hline \multirow{2}{*}{ Total amount of rocurunyum } & female & 43 & 78.023 & 32.000 & 4.880 & \multirow{2}{*}{1.350} & \multirow{2}{*}{0.180} \\
\hline & male & 47 & 69.787 & 25.770 & 3.759 & & \\
\hline
\end{tabular}

*statistically significant $(p<0.05)$; **statistically significant $(p<0.01)$; ***statistically significant $(p<0.001)$ 
Table 2 Descriptive statistics and comparison results for TOF according to genders and periods

\begin{tabular}{|c|c|c|c|c|c|c|c|c|c|c|c|c|c|}
\hline Period & Sex & $n$ & Mean & $\begin{array}{l}\text { Std. } \\
\text { Dev. }\end{array}$ & $\begin{array}{l}\text { Std. } \\
\text { Error }\end{array}$ & t-Value & P-Value & $\mathbf{n}$ & Mean & $\begin{array}{l}\text { Std. } \\
\text { Dev. }\end{array}$ & $\begin{array}{l}\text { Std. } \\
\text { Error }\end{array}$ & F-Value & P-Value \\
\hline \multirow[t]{2}{*}{ TOFO } & female & 43 & 9.535 & 1.882 & 0.287 & \multirow[b]{2}{*}{-0.041} & \multirow[b]{2}{*}{0.967} & \multirow[b]{2}{*}{90} & \multirow[b]{2}{*}{$9.544 \mathrm{~B}$} & \multirow[b]{2}{*}{2.11} & \multirow[b]{2}{*}{0.222} & & \\
\hline & male & 47 & 9.553 & 2.32 & 0.338 & & & & & & & & \\
\hline \multirow[t]{2}{*}{ TOF 25} & female & 43 & 10.628 & 3.2 & 0.488 & \multirow{2}{*}{1.09} & \multirow{2}{*}{0.279} & \multirow{2}{*}{90} & \multirow{2}{*}{$10.256 \mathrm{AB}$} & \multirow{2}{*}{3.103} & \multirow{2}{*}{0.327} & & \\
\hline & male & 47 & 9.915 & 3.006 & 0.438 & & & & & & & & \\
\hline \multirow[t]{2}{*}{$\begin{array}{l}\text { TOF } \\
25-90\end{array}$} & female & 43 & 10.349 & 2.636 & 0.402 & \multirow{2}{*}{-0.44} & \multirow{2}{*}{0.661} & \multirow{2}{*}{90} & \multirow{2}{*}{$10.500 \mathrm{~A}$} & \multirow{2}{*}{3.106} & \multirow{2}{*}{0.327} & 9.034 & $0.000 * * *$ \\
\hline & male & 47 & 10.638 & 3.504 & 0.511 & & & & & & & & \\
\hline \multirow[t]{2}{*}{ TOF end } & female & 43 & I I.605 & 3.417 & 0.521 & \multirow{2}{*}{1.064} & \multirow{2}{*}{0.29} & \multirow{2}{*}{90} & \multirow{2}{*}{ II.I78A } & \multirow{2}{*}{3.643} & \multirow{2}{*}{0.384} & & \\
\hline & male & 47 & 10.787 & 3.833 & 0.559 & & & & & & & & \\
\hline
\end{tabular}

****statistically significant according to One-way Repeated ANOVA $(p<0.00 \mathrm{I})$

Means that do not share a letter are significantly different according to Tukey test $(p<0.05)$

Table 3 Descriptive statistics and comparison results for TOF according to BMI groups

\begin{tabular}{|c|c|c|c|c|c|c|c|}
\hline & BMIGRP2 & Mean & $\mathbf{n}$ & Std. Dev. & Std. Error & F-Value & P-Value \\
\hline & Healthy $(<25)$ & 8.917 & 24 & 2.062 & 0.421 & & \\
\hline \multirow[t]{3}{*}{ TOF0 } & Overweight (25-30) & 9.633 & 30 & 1.79 & 0.327 & 1.589 & 0.21 \\
\hline & Obese $(>30)$ & 9.889 & 36 & 2.339 & 0.39 & & \\
\hline & Healthy $(<25)$ & 9.75 & 24 & 3.22 & 0.657 & & \\
\hline \multirow[t]{3}{*}{ TOF25 } & Overweight (25-30) & 10.267 & 30 & 2.864 & 0.523 & 0.514 & 0.6 \\
\hline & Obese $(>30)$ & 10.583 & 36 & 3.255 & 0.542 & & \\
\hline & Healthy $(<25)$ & 9.25 & 24 & 2.786 & 0.569 & & \\
\hline \multirow[t]{3}{*}{ TOF25-90 } & Overweight (25-30) & 10.8 & 30 & 2.882 & 0.526 & 2.831 & 0.064 \\
\hline & Obese $(>30)$ & 11.083 & 36 & 3.324 & 0.554 & & \\
\hline & Healthy $(<25)$ & $8.917 B$ & 24 & 3.189 & 0.651 & & \\
\hline \multirow[t]{2}{*}{ TOFend } & Overweight (25-30) & $11.733 \mathrm{AB}$ & 30 & 3.29 & 0.601 & 7.375 & $0.001 * *$ \\
\hline & Obese $(>30)$ & $12.222 \mathrm{~A}$ & 36 & 3.634 & 0.606 & & \\
\hline
\end{tabular}

**statistically significant according to One-way Repeated ANOVA $(p<0.001)$

Means that do not share a letter are significantly different according to Tukey test $(p<0.05)$

Table 4 Descriptive statistics and comparison results for BUN. Bun/Cre ratio. Cre and $\mathrm{CrCl}$ according to the genders and TOF periods

\begin{tabular}{|c|c|c|c|c|c|c|c|c|c|c|c|c|c|c|}
\hline Variables & & Sex & $\mathbf{n}$ & Mean & $\begin{array}{l}\text { Std. } \\
\text { Dev. }\end{array}$ & $\begin{array}{l}\text { Std. } \\
\text { Error }\end{array}$ & t-Value & P-Value & $\mathbf{n}$ & Mean & $\begin{array}{l}\text { Std. } \\
\text { Dev. }\end{array}$ & $\begin{array}{l}\text { Std. } \\
\text { Error }\end{array}$ & t-Value & P-Value \\
\hline \multirow{4}{*}{ BUN } & Pre & female & 43 & 17.116 & 7.162 & 1.092 & 0.049 & 0.961 & 90 & 17.078 & 7.07I & 0.745 & \multirow{3}{*}{2.329} & \multirow{4}{*}{$0.022 *$} \\
\hline & \multirow{3}{*}{ Post } & female & 43 & 16.488 & 5.04 & 0.769 & \multirow{3}{*}{0.59} & \multirow{3}{*}{0.556} & \multirow{3}{*}{90} & \multirow{3}{*}{16.178} & \multirow{3}{*}{4.756} & \multirow{3}{*}{0.501} & & \\
\hline & & & & & & & & & & & & & & \\
\hline & & & 41 & $10.0 \%$ & .311 & & & & & & & & & \\
\hline \multirow{4}{*}{$\begin{array}{l}\text { BUN/Cre } \\
\text { ratio }\end{array}$} & \multirow{2}{*}{ Pre } & female & 43 & 19.29 & 8.85 & 1.35 & \multirow{2}{*}{1.292} & \multirow{2}{*}{0.2} & \multirow{2}{*}{90} & \multirow{2}{*}{18.173} & \multirow{2}{*}{7.872} & \multirow{2}{*}{0.83} & \multirow{4}{*}{0.325} & \multirow{4}{*}{0.746} \\
\hline & & male & 47 & $|7.15|$ & 6.794 & 0.991 & & & & & & & & \\
\hline & \multirow[b]{2}{*}{ Post } & female & 43 & 18.386 & 6.125 & 0.934 & \multirow[b]{2}{*}{0.597} & \multirow[b]{2}{*}{0.552} & \multirow[b]{2}{*}{90} & \multirow[b]{2}{*}{17.99} & \multirow[b]{2}{*}{5.991} & \multirow[b]{2}{*}{0.631} & & \\
\hline & & male & 47 & 17.628 & 5.908 & & & & & & & & & \\
\hline & & female & 43 & 0.961 & 0.448 & 0.068 & & & & & & & & \\
\hline & Pre & male & 47 & 1.07 & 0.644 & 0.094 & -0.928 & 0.356 & 90 & 1.018 & 0.559 & 0.059 & & \\
\hline Cre & & female & 43 & 0.963 & 0.402 & $0.06 \mathrm{~J}$ & & & & & & & 2.595 & $0.011 *$ \\
\hline & Post & & & & & & -0.135 & 0.893 & 90 & 0.97 & 0.475 & 0.05 & & \\
\hline & & male & 47 & 0.976 & 0.538 & 0.078 & & & & & & & & \\
\hline
\end{tabular}


Table continued..

\begin{tabular}{|c|c|c|c|c|c|c|c|c|c|c|c|c|c|c|}
\hline Variables & & Sex & $\mathbf{n}$ & Mean & $\begin{array}{l}\text { Std. } \\
\text { Dev. }\end{array}$ & $\begin{array}{l}\text { Std. } \\
\text { Error }\end{array}$ & t-Value & P-Value & $\mathbf{n}$ & Mean & $\begin{array}{l}\text { Std. } \\
\text { Dev. }\end{array}$ & $\begin{array}{l}\text { Std. } \\
\text { Error }\end{array}$ & t-Value & P-Value \\
\hline \multirow{2}{*}{$\mathrm{CrCl}$} & Pre & $\begin{array}{l}\text { female } \\
\text { male }\end{array}$ & $\begin{array}{l}43 \\
47\end{array}$ & $\begin{array}{l}100.35 \\
123.33\end{array}$ & $\begin{array}{l}44.974 \\
46.672\end{array}$ & $\begin{array}{l}6.859 \\
6.808\end{array}$ & -2.375 & $0.020 *$ & 90 & I I 2.357 & 47.05 & 4.96 & \multirow{2}{*}{-1.9} & \multirow{2}{*}{0.061} \\
\hline & Post & $\begin{array}{l}\text { female } \\
\text { male }\end{array}$ & $\begin{array}{l}43 \\
47\end{array}$ & $\begin{array}{l}99.216 \\
131.406\end{array}$ & $\begin{array}{l}46.85 \\
47.634\end{array}$ & $\begin{array}{l}7.145 \\
6.948\end{array}$ & -3.228 & $0.002 * *$ & 90 & I I 6.027 & 49.699 & 5.239 & & \\
\hline
\end{tabular}

Table 5 Descriptive statistics and comparison results for BUN. BUN/Cre ratio. Cre and $\mathrm{CrCl}$ according to the BMI groups

\begin{tabular}{|c|c|c|c|c|c|c|c|c|}
\hline & & BMIGRP2 & $\mathbf{n}$ & Mean & Std. Dev. & Std. Error & F-Value & P-Value \\
\hline \multirow{6}{*}{ BUN } & \multirow{3}{*}{ Preop } & Healthy $(<25)$ & 24 & 14.708 & 6.49066 & 1.3249 & \multirow{3}{*}{1.891} & \multirow{3}{*}{0.157} \\
\hline & & Overweight(25-30) & 30 & 17.767 & 7.17122 & 1.30928 & & \\
\hline & & Obese $(>30)$ & 36 & 18.083 & 7.18083 & I.1968 & & \\
\hline & \multirow{3}{*}{ Postop } & Healthy $(<25)$ & 24 & 14.333 & 4.51728 & 0.92209 & \multirow{3}{*}{2.576} & \multirow{3}{*}{0.082} \\
\hline & & Overweight (25-30) & 30 & 16.7 & 4.90707 & 0.8959 & & \\
\hline & & Obese $(>30)$ & 36 & 16.972 & 4.576 & 0.763 & & \\
\hline \multirow{6}{*}{ BUN/Cre ratio } & \multirow{3}{*}{ Preop } & Healthy $(<25)$ & 24 & 16.774 & 6.273 & 1.28 & \multirow{3}{*}{0.64} & \multirow{3}{*}{0.53} \\
\hline & & Overweight (25-30) & 30 & 18.143 & 7.659 & 1.398 & & \\
\hline & & Obese $(>30)$ & 36 & 19.13 & 8.991 & 1.499 & & \\
\hline & \multirow{3}{*}{ Postop } & Healthy $(<25)$ & 24 & 17.557 & 5.576 & 1.138 & \multirow{3}{*}{0.115} & \multirow{3}{*}{0.891} \\
\hline & & Overweight (25-30) & 30 & 17.943 & 6.27 & $\mathrm{I} .145$ & & \\
\hline & & Obese $(>30)$ & 36 & 18.317 & 6.165 & 1.028 & & \\
\hline \multirow{6}{*}{ Cre } & \multirow{3}{*}{ Preop } & Healthy $(<25)$ & 24 & 0.905 & 0.353 & 0.072 & \multirow{3}{*}{0.829} & \multirow{3}{*}{0.44} \\
\hline & & Overweight (25-30) & 30 & 1.103 & 0.776 & 0.142 & & \\
\hline & & Obese $(>30)$ & 36 & 1.022 & 0.446 & 0.074 & & \\
\hline & \multirow{3}{*}{ Postop } & Healthy $(<25)$ & 24 & 0.856 & 0.323 & 0.066 & \multirow{3}{*}{0.953} & \multirow{3}{*}{0.389} \\
\hline & & Overweight (25-30) & 30 & 1.023 & 0.63 & 0.115 & & \\
\hline & & Obese $(>30)$ & 36 & 1.001 & 0.405 & 0.067 & & \\
\hline \multirow{6}{*}{$\mathrm{CrCl}$} & \multirow{6}{*}{ Postop } & Healthy $(<25)$ & 24 & 101.835 & 38.237 & 7.805 & \multirow{3}{*}{1.076} & \multirow{3}{*}{0.345} \\
\hline & & Overweight(25-30) & 30 & III.642 & 44.689 & 8.159 & & \\
\hline & & Obese $(>30)$ & 36 & 119.968 & 53.644 & 8.941 & & \\
\hline & & Healthy $(<25)$ & 24 & 106.355 & 38.995 & 7.96 & \multirow{3}{*}{0.616} & \multirow{3}{*}{0.543} \\
\hline & & Overweight (25-30) & 30 & 119.215 & 50.519 & 9.223 & & \\
\hline & & Obese $(>30)$ & 36 & 119.818 & 55.492 & 9.249 & & \\
\hline
\end{tabular}

Table 6 Correlation matrix between TOF variables and preoperative and postoperative renal variables

\begin{tabular}{|c|c|c|c|c|c|c|c|c|c|c|c|c|c|}
\hline & $\begin{array}{l}\text { TOF- } \\
25\end{array}$ & $\begin{array}{l}\text { TOF- } \\
25-90\end{array}$ & $\begin{array}{l}\text { TOF } \\
\text { SON }\end{array}$ & $\begin{array}{l}\text { Total- } \\
\text { Roc }\end{array}$ & $\begin{array}{l}\text { Bun- } \\
\text { pre }\end{array}$ & $\begin{array}{l}\text { Bun- } \\
\text { post }\end{array}$ & $\begin{array}{l}\text { BCR } \\
\text { pre }\end{array}$ & $\begin{array}{l}\text { BCR } \\
\text { post }\end{array}$ & $\begin{array}{l}\text { Cre } \\
\text { pre }\end{array}$ & $\begin{array}{l}\text { Cre } \\
\text { post }\end{array}$ & $\begin{array}{l}\text { CrCL } \\
\text { pre }\end{array}$ & $\begin{array}{l}\text { CrCL } \\
\text { post }\end{array}$ & TOF-0 \\
\hline & 0.129 & & & & & & & & & & & & \\
\hline \multirow{2}{*}{ TOF-25 } & 0.226 & & & & & & & & & & & & \\
\hline & 0.056 & 0.457 & & & & & & & & & & & \\
\hline \multirow{2}{*}{$\begin{array}{l}\text { TOF-25- } \\
90\end{array}$} & 0.597 & $0.000 * * *$ & & & & & & & & & & & \\
\hline & 0.158 & 0.426 & 0.594 & & & & & & & & & & \\
\hline \multirow{2}{*}{ TOFend } & 0.137 & $0.000 * * *$ & $0.000 * * *$ & & & & & & & & & & \\
\hline & 0.394 & 0.397 & 0.503 & 0.714 & & & & & & & & & \\
\hline
\end{tabular}


Table continued..

\begin{tabular}{|c|c|c|c|c|c|c|c|c|c|c|c|c|c|}
\hline & $\begin{array}{l}\text { TOF- } \\
25\end{array}$ & $\begin{array}{l}\text { TOF- } \\
25-90\end{array}$ & $\begin{array}{l}\text { TOF } \\
\text { SON }\end{array}$ & $\begin{array}{l}\text { Total- } \\
\text { Roc }\end{array}$ & $\begin{array}{l}\text { Bun- } \\
\text { pre }\end{array}$ & $\begin{array}{l}\text { Bun- } \\
\text { post }\end{array}$ & $\begin{array}{l}\text { BCR } \\
\text { pre }\end{array}$ & $\begin{array}{l}\text { BCR } \\
\text { post }\end{array}$ & $\begin{array}{l}\text { Cre } \\
\text { pre }\end{array}$ & $\begin{array}{l}\text { Cre } \\
\text { post }\end{array}$ & $\begin{array}{l}\text { CrCL } \\
\text { pre }\end{array}$ & $\begin{array}{l}\text { CrCL } \\
\text { post }\end{array}$ & TOF-0 \\
\hline TotalRo- & 0.000 **** & $0.000 * * * *$ & $0.000 * * *$ & $0.000 * * *$ & & & & & & & & & \\
\hline curoniu & 0.513 & 0.118 & 0.03 & -0.054 & 0.148 & & & & & & & & \\
\hline BUN & $0.000 * * *$ & 0.269 & 0.78 & 0.614 & 0.163 & & & & & & & & \\
\hline preop & 0.253 & 0.154 & 0.17 & 0.311 & 0.437 & -0.024 & & & & & & & \\
\hline BUN & $0.016^{*}$ & 0.146 & 0.11 & $0.003^{* *}$ & $0.000 * * *$ & 0.825 & & & & & & & \\
\hline postop & 0.285 & 0.197 & 0.209 & 0.304 & 0.384 & 0.012 & 0.88 & & & & & & \\
\hline $\mathrm{BUN} / \mathrm{Cr}$ & $0.006 * *$ & 0.062 & $0.049 *$ & $0.004 * *$ & $0.000 * * *$ & 0.909 & $0.000 * * *$ & & & & & & \\
\hline preop & 0.131 & -0.22 & -0.218 & -0.211 & -0.03 & 0.045 & 0.536 & 0.435 & & & & & \\
\hline $\mathrm{BUN} / \mathrm{Cr}$ & 0.218 & $0.038^{*}$ & $0.039 *$ & $0.046^{*}$ & 0.781 & 0.673 & $0.000 * * *$ & $0.000 * * * *$ & & & & & \\
\hline postop & 0.148 & -0.227 & -0.236 & -0.322 & -0.198 & 0.104 & 0.242 & 0.33 & 0.735 & & & & \\
\hline Cre & 0.165 & $0.031^{*}$ & $0.025^{*}$ & $0.002 * *$ & 0.061 & 0.33 & $0.022 *$ & $0.001 * *$ & $0.000 * * *$ & & & & \\
\hline preop & 0.156 & 0.354 & 0.332 & 0.542 & 0.519 & -0.032 & 0.54 & 0.453 & -0.326 & -0.421 & & & \\
\hline Cre & 0.143 & $0.00 I^{*}$ & $0.00 I^{* *}$ & $0.000 * * * * *$ & $0.000^{* *}$ & 0.766 & $0.000 * * *$ & $0.000 * * *$ & $0.002^{* *}$ & $0.000^{* * *}$ & & & \\
\hline postop & 0.107 & 0.37 & 0.37 & 0.557 & 0.535 & -0.041 & 0.545 & 0.478 & -0.291 & -0.512 & 0.956 & & \\
\hline $\mathrm{CrCl}$ & 0.314 & $0.000 * * *$ & $0.000 * * *$ & $0.000 * * *$ & $0.000 * * *$ & 0.698 & $0.000 * * *$ & $0.000 * * *$ & $0.005^{* *}$ & $0.000^{* * *}$ & $0.000 * * *$ & & \\
\hline preop & -0.247 & -0.193 & -0.285 & -0.302 & -0.383 & 0.116 & -0.54 & -0.559 & 0.105 & 0.183 & -0.642 & -0.638 & \\
\hline $\mathrm{CrCl}$ & $0.019^{*}$ & 0.069 & $0.006 * *$ & $0.004 * *$ & $0.000 * * *$ & 0.276 & $0.000 * * *$ & $0.000 * * *$ & 0.326 & 0.084 & $0.000 * * *$ & $0.000 * * *$ & \\
\hline postop & -0.2 & -0.202 & -0.315 & -0.323 & -0.376 & 0.081 & -0.501 & -0.518 & 0.056 & 0.289 & -0.564 & -0.647 & 0.93 \\
\hline TOF-0 & 0.059 & 0.056 & $0.003^{* *}$ & $0.002 * *$ & $0.000 * * * *$ & 0.445 & $0.000 * * *$ & $0.000 * * *$ & 0.603 & $0.006^{* *}$ & $0.000 * * *$ & $0.000 * * * *$ & $0.000 * * *$ \\
\hline
\end{tabular}

r, Pearson correlation coefficient

*statistically significant $(\mathrm{p}<0.05)$; **statistically significant $(\mathrm{p}<0.0 \mathrm{I})$; ***statistically significant $(\mathrm{p}<0.00 \mathrm{I})$

\section{Discussion}

In the present study, we investigated the effect of creatinine clearance $(\mathrm{CrCl})$ and $\mathrm{BUN} / \mathrm{Cre}$ ratio in reversal of non-depolarizing blockade with sugammadex. We found that the reverse- acting potency of sugammadex was not affected by body mass index (BMI), or did not differ according to gender. TOF measurements were not affected by preoperative or postoperative BUN and BUN/Cre ratio but showed strong correlation with preoperative and postoperative Cre and $\mathrm{CrCl}$ values. Therefore, it was concluded that creatinine clearance and $\mathrm{BUN} / \mathrm{Cre}$ ratio showed minimal effect on reversing potential of non-depolarizing blockade with sugammadex.

Persistence of the effect of muscle relaxing agents administered under general anesthesia at the postoperative period is called postoperative residual curarization (PORC) (6). Studies report the incidence of PORC between $20-41 \% .^{6-8}$ PORC can lead to complications such as apnea, atelectasis, hypoxia and pneumonia. This condition causes increased morbidity and mortality, resulting in prolonged hospital stay and increased opération costs. For these reasons, in order to prevent PORC, it is recommended to administer short and medium-acting muscle relaxants, to reverse the action of neuromuscular blocking agents at the end of the operation and to follow-up patients with neuromuscular monitoring. ${ }^{6-8}$ Sugammadex is now used routinely in the general anesthesia practice for reversal of neuromuscular blockade $(9,10)$. The reason why sugammadex is preferred is that it is capable of reversing neuromuscular blockade of any depth effectively and rapidly before spontaneous reversal occurs ${ }^{9}$

Xue et al. examined the effect of gender on rocuronium and they reported that females were more sensitive to rocuronium compared to males, resulting in $30 \%$ less rocuronium requirement to achieve the same level of neuromuscular blocking action. ${ }^{11}$ In that study, despite using the same rocuronium dose during induction, time to reach TOF25 was found to be longer among females. Mencke et al. examined the effect of gender on pharmacodynamic actions of rocuronium and they reported that following administration of 0.45 $\mathrm{mg} / \mathrm{kg}$ rocuronium under general anesthesia, the time to reach TOF0 after induction was shorter while time to reach TOF25 was longer among female patients. ${ }^{12}$ In the same study, no difference was found between males and females regarding recovery times after operation. ${ }^{12}$ In our study, we compared time to reach TOF0 and TOF25 between male and female patients following administration of equal dose of i.v. rocuronium per $\mathrm{kg}$ of body weight $(0.6 \mathrm{mg} / \mathrm{kg})$ under general anesthesia with sevoflurane. There was no difference with regard to mean patient age and operating room temperature was standardized. $2 \%$ sevoflurane was applied as standard. According to our results, there was no statistically significant difference regarding mean TOF measurements between male and female patients in all periods $(\mathrm{p}>0.05)$. Our results are consistent with previously reported findings.

The elimination of sugammadex or sugammadex-rocuronium complex is significantly prolonged in patients with severe renal damage $(\mathrm{CrCl}<30 \mathrm{ml} / \mathrm{min})$ when compared to patients with normal renal 
function $(\mathrm{CrCl}>80 \mathrm{ml} / \mathrm{min})$. However, no alteration was observed in the efficacy of sugammadex, or no neuromuscular re-curarization was observed (1). One study compared sugammadex and neostigmine for their short-term effects on glomerular filtration and tubular function in patients with normal renal function and the effect of sugammadex on glomerular and tubular system was found to be more tolerable compared to neostigmine. ${ }^{9}$ Another study found delayed excretion of sugammadex and sugammadex-rocuronium complex in patients with severe renal failure but did not detect neuromuscular recurarization. ${ }^{13}$ Studies in literature did not find significant difference between patients with normal renal function and patients with severe renal damage in terms of the efficacy of sugammadex in reversal of neuromuscular blockade caused by rocuronium. Time to reach TOF90 with $2 \mathrm{mg} / \mathrm{kg}$ sugammadex is 1.6 minutes in patients with normal renal function, while this time is 2 minutes in patients with severe renal damage. ${ }^{1}$ In agreement with the literature, in our study, t-test results for TOF did not show statistically significant difference in mean values between patient groups in all periods ( $p>0.05$ ). The difference between TOF periods was compared with repeated measures analysis of variance and accordingly, the difference between periods was found to be statistically significant $(\mathrm{p}<0.001)$. Tukey test results did not show difference between TOF25, TOF25-90 and TOFend times, while initial TOF value was found to be significantly lower in comparison to TOF25-90 and final TOF time ( $<<0.05)$. We think this is related to the fact that rocuronium dose was calculated according to their real body weight rather than ideal body weight.

One rat study showed that high dose sugammadex administration caused profound histopathological degeneration in renal tissues (glomerular vacuolization, tubular dilation, vascular vacuolization and hypertrophy, lymphocyte infiltration). The same study emphasized that increasing doses of sugammadex can be associated with increased histopathological alteration in kidneys. The study found more profound histopathological changes in the group that received only rocuronium compared to the group that received only sugammadex, therefore the authors suggested that sugammadex had mild effect on renal cells and could cause greater histopathological degeneration when combined with rocuronium. The authors did not find significant difference with regard to BUN, Cre, sodium and potassium concentrations measured as part of renal function tests. ${ }^{14}$ Unlike the study by Bostan et al. in our study, however, comparison of BUN, BUN/Cre ratio, Cre and $\mathrm{CrCl}$ values between preoperative and postoperative period showed moderate improvement in renal functions. These findings suggest that sugammadex can have beneficial effects on renal functions.

Preoperative dehydration is one of the causes of increased postoperative morbidity. One study showed that postoperative complications are observed 4 times more frequent in dehydrated patients compared to hydrated patients. ${ }^{15}$ Preoperative dehydration is quite prevalent (33\%), but it is rarely serious. ${ }^{15}$ Increased BUN/Cre ratio is regarded as a sign of dehydration and studies suggest that it can predict mortality especially in certain disease groups and result in increased mortality ${ }^{17,18}$ Lin et al. examined perioperative BUN/Cre ratio in gastrointestinal cancer patients with enteric fistula and found that increased BUN/Cre ratio was associated with increased mortality in this patient group..$^{19}$ In the literature, there is no study examining the association between BUN/Cre ratio and TOF. Therefore, our study is the first study in this respect. In our study, we found very strong negative correlation with TOF0 values in patients with increased preoperative and postoperative BUN/Cre ratio (with BUN Cre ratio 20 or higher). As BUN/Cre ratio increases, time to reach TOF0 decreases. Therefore, rocuronium dose should be kept low in dehydrated patients with increased BUN/Cre ratio.

Studies related to administration of neuromuscular blocking agents according to BMI showed that it would be more appropriate to administer neuromuscular blocking agents based on IBW in obese patients and that neuromuscular blockade action can be prolonged in such patients if these agents are administered based on the real body weight. ${ }^{20}$ In our study, when we examined TOF measurement analyses performed according to BMI, TOF end was found to be higher than the rest of measurement times ( $\mathrm{p}=0.001)$. In obese patients $(\mathrm{BMI}>30)$, TOF end time was profoundly prolonged. We think this is because we administered rocuronium doses based on patient's real body weight. In obese patients, especially the lipophilic drugs are known to have different volume of distribution between body's fat and fluid compartments. ${ }^{21,22}$ This led to calculation of anesthetic agents according to ideal body weight in especially obese patients. In their study, Sanflippo et al. administered sugammadex dose adjusted according to IBW or real body weight and did not find difference between the two methods regarding recovery time or PORC. ${ }^{23}$ On the other hand, in our study, we found that TOF end was prolonged because of administration of sugammadex based on patient's real body weight; however, we did not observe PORC in any of our cases. Our results showed that administration of sugammadex based on patient's current body weight did not have any adverse effect on kidney functions. Our results are consistent with the literature findings.

\section{Conclusion}

In conclusion, the reverse acting potency of sugammadex was unaltered in cases with impaired kidney function with reduced creatinine clearance and as well as in dehydrated cases with increased $\mathrm{BUN} / \mathrm{Cre}$ ratio. Therefore, we believe it can be used safely. In addition, considering that obesity surgery is popular around the whole world, sugammadex can be safely used in recurarization of obese patients. Sugammadex should be used in both obese patients with impaired kidney function in order to minimize PORC risk and TOF monitoring should become a routine practice.

\section{Ethics committee approval}

Clinical Studies Ethics Committee of Ordu University, Faculty of Medicine and June 2017 (Issue date 24/08/2017, Issue number: 2017/93).

\section{Informed consent}

Written informed consent was obtained from patients who participated in this study.

\section{Financial disclosure}

The authors declare no financial support.

\section{Details authors contribution}

${ }^{1}$ Ebru Canakci: Concept, study design, literature rewiev, write the manuscript, data analyze, data collection and final review

${ }^{2}$ Ahmet Karatas: Concept, study design, conducts the study, data collection, analyze the data, literature rewiev, and write the manuscript 


\section{Acknowledgements}

This article abstract was presented in Congress of National 35th Nephrology, Dialysis, Hypertension and Transplantation, 3-7 October, Antalya, Turkey.

\section{Conflict of interest}

No conflict of interest was declared by the authors.

\section{References}

1. Staals LM, Snoeck MM, Driessen JJ, et al. Multicentre, parallel-group, comparative trial evaluating the efficacy and safety of sugammadex in patients with end-stage renal failure or normal renal function. $\mathrm{Br} J$ Anaesth. 2008;101(4):492-497.

2. Staals LM, Snoeck MM, Driessen JJ, et al. Reduced clearance of rocuronium and sugammadex in patients with severe to end-stage renal failure: a pharmacokinetic study. Br J Anaesth. 2010;104(1):31-39.

3. Yang LP, Keam SJ. Sugammadex: a review of its use in anesthetic practice. Drugs. 2009;69(7):919-942.

4. Parlakpınar H, Orum MH, Acet A. Free Oxygen Radicals in Drug-Induced Nephrotoxicity. FU Săg. Bil Vet Derg. 2013;27(1):51-56.

5. Set T, Sahin ME. Kidney Function Tests For Primary Healthcare Physician. Sted. 2003;12(9):344-345.

6. Naguib M, Kopman AF, Ensor JE. Neuromuscular monitoring and postoperative residual curarisation: a meta-analysis. $\mathrm{Br} J$ Anaesth. 2007;98(3):302-316.

7. Booij LH. Cyclodextrins and the emergence of sugammadex. Anaesthesia. 2009;64(1):31-37.

8. Claudius C, Garvey LH, Viby-Mogensen J. The undesirable effects of neuromuscular bloking drugs. Anaesthesia. 2009;64(1):10-21.

9. Fuchs Buder T, Meistelman C, Raft J. Sugammadex: clinical development and practical use. Korean J Anesthesiol. 2013;65(6):495-500.

10. Pongrácz A, Szatmári S, Nemes R, et al. Reversal of neuromuscular blockade with sugammadex at the reappearance of four twitches TrainOf-Four Stimulation. Anesthesiology. 2013;119(1):36-42.

11. Xue FS, Liao X, Liu JH, et al. Dose-response curve and time-course of effect of vecuronium in male and female patients. $\mathrm{Br} J$ Anaesth. 1998;80(6):720-724
12. Mencke T, Soltész S, Grundmann U, et al. Time course of neuromuscular blockade after rocuronium. Anaesthesist. 2000;49(7):609-612.

13. Adam JM, Bennett DJ, Bom A, et al. Cyclodextrin-derived hos molecules as reversal agents for the neuromuscular blocker rocuronium bromide: synthesis and structure-activity relationships. J Med Chem. 2002;45(9):1806-1816.

14. Bostan H, Kalkan Y, Tomak Y, et al. Reversal of rocuronium-induced neuromuscular block with sugammadex and resulting histopathological effects in rat kidneys. Ren Fail. 2011;33(10):1019-1024.

15. Ylinenvaara SI, Elisson O, Berg K, et al. Preoperative urine-specific gravity and the incidence of complications after hip fracture surgery: A prospective, observational study. Eur J Anaesthesiol. 2014;31(2):85-90.

16. Hahn RG, Waldréus N. An Aggregate Urine Analysis Tool to Detect Acute Dehydration. Int J Sport Nutr Exerc Metab. 2013;23(4):303-311.

17. Brisco MA, Zile MR, Ter Maaten JM, et al. The risk of death associated with proteinuria in heart failure is restricted to patients with elevated blood urea nitrogen to creatinine ratio. Int J Cardiol. 2016;215(1):521-526.

18. Lin HJ, Chao CL, Chien KL, et al. Elevated blood urea nitrogen-tocreatinine ratio increased the risk of hospitalization and all-cause death in patients with chronic heart failure. Clin Res Cardiol. 2009;98(8):487-492.

19. Lin $\mathrm{LH}$, Chen CW, Lu CY, et al. High preoperative ratio of blood urea nitrogen to creatinine increased mortality in gastrointestinal cancer patients who developed postoperative enteric fistulas. Kaohsiung $\mathrm{J} \mathrm{Med}$ Sci. 2012;28(8):418-422.

20. Leykin Y, Pellis T, Lucca M, et al. The Pharmacodynamic Effects of Rocuronium When Dosed According to Real Body Weight or Ideal Body Weight in Morbidly Obese Patients. Anesth Analg. 2004;99(4):1086 -1089 .

21. Apfelbaum JL, Grasela TH, Hug CC Jr, et al. The initial clinical experience of 1819 physicians in maintaining anesthesia with propofol: characteristics associated with prolonged time to awakening. Anesth Analg. 1993;77(4):10-14

22. Gear RW, Miaskowski C, Gordon NC, et al. The kappa opioid nalbuphine produces gender- and dose-dependent analgesia and antianalgesia in patients with postoperative pain. Pain. 1999;83(2):339-345.

23. Sanfilippo M, Alessandri F, Wefki Abdelgawwad Shousha AA, et al. Sugammadex and ideal body weight in bariatric surgery. Anesthesiol Res Pract. 2013;389782. 